\title{
La Real Academia de San Fernando y la construcción de un campo de las Bellas Artes en España en el siglo XVIII. Una panorámica política, social y artística
}

The Royal Academy of San Fernando and the construction of a field of Fine Arts in Spain in the Eighteenth century. Political, social and artistic panoramic

JaCQues SOUBEYroux Universidad de Saint-Etienne

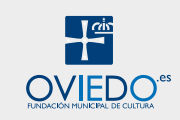




\section{RESUMEN}

Este estudio es una actualización contextual de las investigaciones sobre la Academia de Bellas Artes de Madrid. Moviliza los diferentes conocimientos políticos, económicos, sociales y culturales para mostrar cómo y hasta qué punto la construcción de un campo de Bellas Artes se inscribe en el programa del despotismo ilustrado. Se estudiarán principalmente:

- La dependencia absoluta de la autoridad del monarca,

- el lugar ocupado por las mujeres en la Academia,

- el proyecto de generalización de la enseñanza del dibujo y la pedagogía impuesta por los académicos,

- el papel de la Academia como autoridad artística y su acción por el reconocimiento social de las artes y de los artistas en consonancia con Goya y su obra.

Palabras clave

Bellas Artes, dibujo, despotismo ilustrado, autoridad artística, Goya.

\section{ABSTRACT}

This paper aims to contextually update the research carried out so far on Madrid's Academy of Fine Arts. Using the different knowledge in the cultural, social, economic and political sectors, the paper endeavours to show how and to what extent the creation of a Fine Arts field has been part of the program of enlightened despotism. I will particularly focus on:

— The absolute dependence on the monarch's authority,

— women's role within the Academy,

- the project aimed at generalising the teaching of drawing, and the pedagogy imposed by the members of the Academy,

- the Academy's role as an artistic authority as well as it efforts to improve the social recognition of artists and to support the arts.

KeY WordS

Fine Arts, drawing, enlightened despotism, artistic authority, Gora.

Recibido: 16 de enero de 2020. Aceptado: 22 de junio de 2020. 
Las academias ocupan un lugar aparte en los campos del saber del siglo XVIII español por las relaciones que permiten establecer con sus modelos extranjeros, pero es su inscripción en la realidad española lo que les da su verdadera significación, y esto es particularmente verdadero en el caso de la Academia de Bellas Artes de San Fernando. Si la organización interna de la Academia y sus opciones artísticas quedan suficientemente descritas en los trabajos existentes, éstos señalaron la existencia de factores políticos y socioeconómicos sin analizar verdaderamente el papel que desempeñaron. Y son precisamente estos factores contextuales los que serán el objeto de mi estudio que interrelacionará los aspectos políticos, económicos, sociales y culturales para mostrar hasta qué punto el proyecto de la Academia y su modo de funcionamiento se inscriben en el programa del despotismo ilustrado. Esta puesta al día contextual se apoyará en las informaciones contenidas en la tesis de Claude Bédat ${ }^{1}$ que utilizaré desde un enfoque nuevo, completándolas con otras informaciones que yo mismo recogí en el archivo de la institución madrileña y en mis investigaciones sobre Goya.

\section{Las bases políticas}

Las tres principales academias españolas, de la Lengua, de la Historia y de Bellas Artes se presentan explícitamente como fundaciones reales, nacidas de la voluntad del monarca de crear en España unas instituciones parecidas a las que existían en los países vecinos y que resplandecían en la vida cultural de toda Europa. Sin embargo, la implicación personal de los monarcas es distinta en los tres casos. Sabemos que la iniciativa de la creación de la Real Academia de la Lengua en 1713 se debe al marqués de Villena y que el decreto de aprobación de Felipe V sólo se publicó en octubre de 1714, o sea más de un año después de la primera reunión del equipo de los fundadores. Lo mismo pasó para la Academia de la Historia que existió primero como una «tertulia» reunida

1 Claude Bedat, L'Académie des Beaux-Arts de Madrid (1744-1808), Toulouse, Publications de l'université de Toulouse-Le Mirail, 1974. Existe una versión española, La Real Academia de Bellas Artes de San Fernando (1744-1808). Contribución al estudio de las influencias estilísticas y de la mentalidad artística en la España del siglo XVIII, Madrid, Fundación Universitaria Española, 1989. 
por el abogado de los Reales Consejos, Julián Hermosilla, en 1735, tres años antes de la publicación del decreto de aprobación real de junio de 1738 que la convirtió oficialmente en una «Academia». La génesis de la Academia de Bellas Artes fue todavía más diferente y mucho más larga. Si el proyecto publicado en 1726 por Francisco Antonio Meléndez, pintor de miniaturas, se refería ya a «la fundación de una Academia de Artes en la Corte de Madrid [...] a imitación de las que se hacen ilustres en París, en Florencia y en Flandes»², se debía a una iniciativa personal, ajena a cualquier patronazgo real. En 1744 se fundó una Junta Preparatoria ${ }^{3}$, a iniciativa del escultor italiano Domingo Olivieri quien había creado desde 1741 una academia de escultura que acogía a sus alumnos en los propios apartamentos del escultor, antes de trasladarse a la Casa de la Panadería de la Plaza Mayor en septiembre de 1744. Esta Junta preparatoria, presidida por el marqués de Villarias, había elaborado un proyecto de estatutos que fue aprobado por el rey Felipe V el 13 de julio de 1744. Pero las rivalidades y disensiones dentro de la Asamblea paralizaron su acción y hubo que esperar hasta la llegada al trono de Fernando VI y la intervención personal del nuevo rey para acelerar el proceso de creación de la institución que se realizó en adelante bajo su autoridad de modo que la Academia de Bellas Artes fue la única creada por la iniciativa directa del monarca, de ahí su nombre de San Fernando, como homenaje a su fundador. Él fue quien encargó al escultor Felipe de Castro una revisión del proyecto de los primeros estatutos que fueron aprobados en 1751 y aplicados en la apertura oficial de la Academia en 1752. Él nombró como Protector de la Academia a su ministro de asuntos exteriores José de Carvajal y le pidió que hiciera redactar unos nuevos estatutos, aprobados en $1757^{4}$. Este cambio de estatutos se debe a los conflictos que habían surgido durante este periodo experimental entre los artistas, apegados en su gran mayoría a la estética barroca, y los consiliarios miembros de la élite ilustrada a los que Carlos Sambricio calificó como «representantes de la nueva Razón» ${ }^{5}$.

Castro había incluido en los estatutos de 1751 las principales reivindicaciones de los artistas a quienes se confiaba el gobierno de la nueva institución, como se hizo en los primeros tiempos de la Real Academia de Pintura y Escultura de París cuyo lema era Libertas artibus restituta antes de la toma de control

2 Bedat, L'Académie des Beaux-Arts de Madrid., pág. 3.

3 Véase Esperanza Navarrete Martínez, Catálogo documental de la Junta preparatoria de la Real Academia de Bedllas Artes de San Fernando (1744-1752), Madrid, Real Academia de Bellas Artes de San Fernando, 2007.

4 Estatutos de la Real Academia de San Fernando, en Madrid, en casa de Don Gabriel Ramírez, Impresor de la Real Academia, 1757.

5 Carlos Sambricio, «Las oraciones en la Academia de San Fernando», Revista de ideas estéticas, 136 (1976), págs. 341-366. 
por el poder real en 1665. Los estatutos de la Academia española de 1757 significaban también el dominio completo de la autoridad real: se dividía por dos el número de profesores que pasaban de treinta y uno a dieciséis y se daba el poder a trece consiliarios nobles y veinte «académicos de honor», nombrados todos por el rey. La autoridad real quedaba afirmada con fuerza en los estatutos que precisaban en su artículo XXXI cuál debía ser el origen social de los académicos de honor: «Es mi voluntad que sean de los números de los Grandes, de los Ministros y personas más autorizadas de mi Corte".

Esta dependencia de la autoridad real, subrayada por Sánchez Cantón que habla de un «feudo real gobernado por políticos y aristócratas » ${ }^{6}$, era una novedad importante frente a las demás academias que siempre elegían sus miembros por cooptación y siguen haciéndolo actualmente.

Pero estos consiliarios nombrados por el rey eran a menudo miembros de una o varias otras academias, a pesar del modo de elección distinto de éstas. Era el caso del mismo Protector de la Academia, José de Carvajal, y del Viceprotector Tiburcio Aguirre, y también del duque de Alba, del marqués de Santa Cruz, de Juan y Tomás de Iriarte, de Vicente García de la Huerta y Diego Rejón de Silva, que pertenecieron todos a la Academia de la Lengua, mientras que Antonio Ponz formaba parte de la Academia de la Historia. Por fin Agustín de Montiano, Ignacio de Hermosilla, el duque de Almodóvar, los condes de Torrepalma y de la Roca, Ignacio Luzán y Jovellanos fueron todos miembros de las tres academias, y además algunos de ellos lo fueron también de diferentes sociedades Económicas, Vascongada, Matritense, u otra que pudo cooptarlos.

Estas características hacen de la Real Academia de Bellas Artes una institución emblemática del sistema político denominado, de manera discutible quizás, «despotismo ilustrado». Tal sistema, impulsado precisamente con la llegada al trono de Fernando VI, que coincidió con la creación de la Academia de San Fernando, se caracterizaba por el régimen de «los ministros con el rey » que fue descrito por José Luis Gómez Urdáñez en sus trabajos sobre el marqués de La Ensenada y Fernando VI ${ }^{7}$. El ejemplo de la Academia de San Fernando nos muestra que tal régimen no se aplicaba sólo a la cumbre del Estado, sino que se apoyaba en una red formada por miembros de la élite ilustrada, que pertenecían

6 Francisco Javier Sánchez Cantón, Antonio Rafael Mengs, 1728-1779. Noticia de su vida y de sus obras, citado por BEDAT, L'Académie des Beaux-Arts de Madrid, pág. XIV. Sobre la cuestión de la nobleza, véase Esperanza Navarrete Martínez, «El Archivo de la Real Academia de Bellas Artes de San Fernando de Madrid, y algunas notas sobre la nobleza», en Fuentes de la historiografía madrileña, Madrid, Real Cuerpo de la Nobleza de Madrid, 2009, págs. 79-109.

7 José Luis Gómez Urdáñez, El proyecto reformista de Ensenada, Lleida, Milenio, 1996; id., Fernando VI, Madrid, Arlanza, 2001; id., El marqués de la Ensenada. El secretario de todo, Madrid, Punto de vista, 2017. 
en su mayoría a las grandes familias nobles al servicio del poder real, de tal modo que el campo del saber se constituía como un campo del poder. Pero ¿qué política quedaba determinada por esta conjunción?

\section{La inscripción en el contexto socioeconómico}

Para comprender la significación de la creación por el poder real de un espacio «artístico» autónomo, hay que situar los hechos en el contexto de la década de 1750. Los censos de la segunda mitad del siglo reflejan la confusión que reinaba en aquel entonces entre los artistas y los artesanos de la capital:

a) la categoría definida como «arte de escultores y estatuarios» pasa en Madrid de 69 miembros en el catastro de 1757 a 130 en el censo de Godoy de 1797,

b) los arquitectos, reunidos en un primer tiempo en el Catastro de Madrid de 1757 con los maestros de obras, formaban un grupo más numeroso de 252 miembros. Contados separadamente eran todavía 172 a finales del siglo. Esta abundancia se debía sin duda a los privilegios de que gozaba desde el siglo XVII el colegio de arquitectos que pertenecía a la cofradía de Nuestra Señora de Belén. Estos privilegios, aprobados por el Consejo de Castilla, le permitían otorgar a cualquier artesano el título de arquitecto sin examen previo por otra autoridad.

Pero en la década de 1750 se abría una etapa nueva en las relaciones entre la Corona y las artes reflejada en la real orden de 18 junio de 1756 que reconocía la legalidad del «trabajo libre» de los artesanos no agremiados ${ }^{8}$. Las reivindicaciones de los artistas, incluidas por Felipe de Castro en los estatutos de 1751, se inscribían en el contexto de esta desestructuración en curso del sistema económico y social existente encabezada por el Estado. El artículo XXXIII de los estatutos de la Academia de 1757 prolongaba la real orden de 1756 prohibiendo todas las congregaciones y cofradías que quisieran organizar estudios de pintura, escultura o arquitectura, «usurpando los títulos de colegios de arquitectos, Academia de arquitectura y otros parecidos» ${ }^{9}$.

8 Sobre la situación originada por esta real orden en la artesanía madrileña, véase José A. NiETo, Artesanos y mercaderes. Una historia social y económica de Madrid (1450-1850), Madrid, Fundamentos, 2006, pág. 369 .

9 Archivo de la Real Academia de San Fernando (en adelante ARASF), Distribución de los premios, 1784. 
Aunque la cofradía de Nuestra Señora de Belén estaba citada explícitamente en la real orden, los artesanos que pertenecían a ella siguieron resistiendo durante largos años y hubo que esperar las reales órdenes de 1782 y 1785 para que se impusiera la voluntad real y se reconociera la autoridad de la Academia. Una victoria celebrada en el discurso de distribución de los premios de 1784, en el que se afirmaba: «Había resuelto Su Majestad declarar por punto general ser permitido el libre ejercicio de las mismas (artes) sin que los expresados gremios pudiesen embarazarlo» ${ }^{10}$.

El control por el poder de las que se califican como «las tres nobles artes» se justifica por la concepción utilitaria del arte que tenían los ilustrados y que hacía del dibujo «el padre de los oficios prácticos», según la expresión de Campomanes en su Discurso sobre la educación popular de los artesanos ${ }^{11}$. De tal modo que el «arte» del dibujo se convirtió en la base de un verdadero proyecto económico desarrollado por la Academia, que aparecía explícitamente recordado en el discurso de distribución de los premios de 1790:

El dibujo es la fuente y origen del recto modo de pensar para toda gentil y agradable manufactura; y cuando el conocimiento de éste logre en el Reyno toda la extensión que debemos esperar, las obras de nuestros artífices nos causarán agrado y complacencia: los muebles y alhajas de que nos servimos en nuestras casas se harán con perfección y no habrá ningún motivo para buscarlos ni esperarlos mejores de otras partes. El dibujo será brevemente un ramo principal de la instrucción pública en toda la Monarquía ${ }^{12}$.

El dibujo era pues la asignatura básica que debían seguir todos los alumnos y era la misión encargada a la Academia enseñársela para mejorar a medio plazo la calidad de las manufacturas fabricadas en España para que no sea ya necesario importarlas desde el extranjero. De ahí la función principalmente educativa atribuida a la Academia en los estatutos de 1751, del todo comparable con la de las escuelas primarias:

Apartar a la juventud española de la ociosidad, madre de todos los vicios, animándola por el ejemplo, la instrucción y la recompensa a cultivar y propagar las tres nobles artes de la pintura, la escultura y la arquitectura.

10 ARASF, Distribución de los premios, 1790.

11 Pedro Rodríguez de Campomanes, Discurso sobre la educación popular de los artesanos, ed. de John Reeder, Madrid, Clásicos del pensamiento económico, 1975, pág. 56.

12 ARASF, Distribución de los premios, 1790. 
A cambio de esta noble misión de interés nacional, los académicos obtenían el privilegio de la nobleza personal con las prerrogativas e inmunidades de que gozaban los nobles de sangre. Esta disposición figuraba en los estatutos de 1751 y Felipe de Castro la había justificado citando el ejemplo de Velázquez que había sido ennoblecido por Felipe IV. El artículo XXXIV de los estatutos de 1757 confirmaba esta medida que iba mucho más allá del prestigio que se suponía que iba a dar a la institución y a los mismos académicos: el ennoblecimiento de los «artistas», que creaba una distancia esencial con los artesanos, desestructuraba en realidad toda la jerarquía social existente creando una categoría nueva de nobles activos que habían de ser los motores del progreso, un esquema que se volverá a encontrar en numerosos proyectos de reforma de las Sociedades Económicas. Sin embargo, las encuestas genealógicas que emprendieron varios académicos, entre ellos el mismo Goya, para probar el origen noble de su linaje muestran que no les parecía suficiente esa forma de nobleza personal que no les daba un prestigio comparable con el de la nobleza hereditaria a la que pertenecían la mayoría de los consiliarios. Privilegiados frente al mundo de los artesanos exteriores a la Academia, los profesores se sentían todavía inferiores a los aristócratas que regentaban la institución y con los cuales mantenían relaciones tensas.

El lugar ocupado por las mujeres era otro problema importante en la reestructuración social iniciada por el gobierno y ahí también la Academia de San Fernando se mostró más abierta que las demás instituciones de su tiempo. Entre los académicos de honor — que debían pertenecer todos a las familias de los Grandes- había no sólo hombres, sino también mujeres: las marquesas de Estepa, de Santa Cruz, de Villafranca, así como las hijas del marqués de Yebra y del conde de Montijo. De modo que en 1772 no había menos de doce académicas de honor. Entre ellas el ejemplo más emblemático es sin duda el de la duquesa de Huéscar, hija del marqués de Santa Cruz (y madre de la duquesa de Alba retratada por Goya), que mandó en 1766 un dibujo de su mano merecedor de elogios ditirámbicos de los académicos. No sólo fue nombrada académica de honor y Directora honoraria de la sección Pintura, sino que tuvo el privilegio de ser la única mujer autorizada a participar con derecho de voto y asiento de honor en las reuniones de la Junta de gobierno de la Academia, que habían sido exclusivamente masculinas hasta entonces ${ }^{13}$.

13 ARASF, Libro 3/82, Junta ordinaria de 20 de julio de 1766. Sobre el caso de la duquesa de Huéscar, véase también Francisco Agullar Piñal, Madrid en tiempos del «mejor alcalde», San Cugat, Arpegio, vol. 4, pág. 200. Sobre el colectivo de las mujeres académicas, véase el estudio general de Estrella de Diego, La mujer y la pintura del XIX español (Cuatrocientas olvidadas y algunas más), Madrid, Cátedra, 1987. 
Estos ejemplos confirman la estrecha relación que existía entre la aristocracia y la Academia, pero también muestra que ésta iba por delante de las demás instituciones en cuanto a la admisión de las mujeres. Esta situación fue citada como un precedente por Manuel Josef Marín en la memoria que leyó el 28 de octubre de 1775 en la apertura del debate en la Matritense, donde las mujeres no fueron admitidas hasta 1786 . Con ser un caso excepcional, el privilegio otorgado a la duquesa de Huéscar en 1766 iba mucho más allá de las propuestas de Marín y de Campomanes en sus discursos de octubre y noviembre de 1775, que sólo preveían para las mujeres una situación de miembro asociado ${ }^{14}$.

A las aristócratas nombradas académicas de honor se añadían «académicas de mérito» que debían presentar un expediente de candidatura y una obra personal para ser admitidas (artículo XXXII de los estatutos). Este título se atribuyó a las hijas de varios profesores (Ana María Mengs, Mariana Sabatini, Dorotea Michel), pero también a artistas extranjeras como la italiana Catalina Cherubini y la francesa Marie-Josèphe Carron, conforme a la apertura internacional prevista por los estatutos.

Estos mismos estatutos permitían también admitir a candidatos y candidatas cuyas obras no poseían «toda la perfección necesaria» con el título de «académicos supernumerarios» que las autorizaba para enseñar el dibujo a los niños. A esta categoría pertenecieron dos madrileñas de nivel económico modesto, Bárbara María Hueva y Ana Meléndez ${ }^{15}$. Ésta había presentado en 1759 como prueba de examen una miniatura que representaba a los reyes Fernando VI y María Bárbara que mereció la siguiente valoración:

La Junta, habiendo reconocido este cuadrito, sin embargo de no hallar en él perfección alguna, atendiendo ser muy loable la aplicación en estas artes de las señoras, para animarlas y no desairar a la presente, la declaró por todos los votos Académico de mérito supernumerario ${ }^{16}$.

La alabanza de la práctica de las artes por las mujeres que concluye este juicio condescendiente es recurrente en los discursos de distribución de premios como en los tratados de economía política, de Bernardo Ward a Campomanes,

14 Acerca del debate sobre la admisión de las mujeres en la Matritense, véase Lucienne Domergue, Jovellanos à la Société Economique des Amis du Pays de Madrid (1778-1795), Toulouse, France-Ibérie Recherche, 1971, págs. 240 y sigs, y apéndice XI, págs. 340-343.

15 Ana Meléndez (1717-1782) era hija de Francisco Antonio Meléndez, que había presentado el primer proyecto de creación de la academia, y hermana de los pintores Luis Egidio y Agustín, lo que explica su vinculación con la institución.

16 ARASF, Libro 3/82, Junta ordinaria de 2 de septiembre de 1759. 
que abogan por la integración de las mujeres en el mundo laboral. El ejemplo de la Academia nos muestra cómo podía hacerse esta integración empezando por las aristócratas que servirían de modelo para las clases medias.

En el grupo de los alumnos estaba muy claramente definida la diferencia entre los sexos en el siglo XvIII: las clases de dibujo en las que se proponían reproducciones de modelos desnudos estuvieron reservadas durante largos años a un público masculino, ya que no se encuentra en el archivo de la Academia ninguna mención de trabajos de alumnas antes de 1804.

A pesar de esta restricción, el balance de la asistencia a las clases que se daban en nocturno, primero en el edificio de la Casa de la Panadería, luego a partir de 1774 en el de la calle de Alcalá, atestigua el éxito que conoció la Academia. Según los cálculos de Claude Bédat ${ }^{17}$, desde 1758 unos 300 alumnos seguían las clases de dibujo. Con la apertura de clases nuevas de geometría y de perspectiva (1766), de anatomía (1767), luego de matemáticas (1768), el alumnado siguió creciendo en las décadas siguientes para ascender hasta 1.079 a finales del siglo, repartido casi igualmente entre dibujo (531) y matemáticas (548).

A los alumnos presentes en la institución madrileña hay que añadir los que resultaban de la fundación en todo el país de numerosas escuelas de dibujo en la segunda mitad del siglo. Estas escuelas se debían a la iniciativa de diferentes instituciones, principalmente las Sociedades Económicas de Amigos del País (en Madrid, Vitoria, Valladolid, Segovia, Oviedo, Santiago, Salamanca, Murcia), pero también Cámaras de comercio (en Barcelona, Burgos), instituciones religiosas (en Córdoba, Toledo, Las Palmas) o simples particulares (en Cádiz, Sevilla, Granada, Gerona, Palma de Mallorca). Habían generado una verdadera red de establecimientos que, sea cual fuera su origen, estaban bajo la autoridad de la Academia madrileña encargada, conforme a sus estatutos, de controlar la enseñanza que se daba en ellas. Esta red de escuelas de dibujo provincianas está poco estudiada hasta ahora, a excepción del País Vasco donde sabemos que, gracias a la acción de la Sociedad Económica Bascongada, se crearon seis escuelas a partir de los años de 1774 en Vitoria, Bilbao, Vergara, San Sebastián, Plasencia y Tolosa, cada una con una o dos especialidades: arquitectura y adorno, pero también marinería, comercio, albañilería y carpintería ${ }^{18}$.

Este desarrollo de la enseñanza del dibujo fue aprobado por Campomanes que señalaba en su Discurso sobre la educación popular de los artesanos (1775) los beneficios que resultaban de la acción de la Academia:

17 Bedat, L'Académie des Beaux-Arts de Madrid, págs. 102-103.

18 Mariano Jiménez Ruiz de Ael, «Las escuelas de dibujo del País Vasco en el siglo XviII: sus modelos académicos», Sancho el sabio, Revista de cultura e investigación vasca, 3 (1993), págs. 149-164. 
La experiencia de nuestros días, desde la erección de la academia de San Fernando, hace evidencia de la utilidad y necesidad del dibujo: a vista del progreso, que todas las artes y oficios adquieren en el Reino por virtud de la enseñanza del diseño, que con utilidad ya se va propagando a otros pueblos por la enseñanza de los grandes maestros, individuos de este ilustre cuerpo y por la imitación de sus excelentes obras.

E insistía en el provecho que sacarían los artesanos de esta enseñanza:

Las artes y oficios, que inmediatamente no necesiten el dibujo, se ven precisadas a valerse de él, para dar a conocer sus instrumentos, máquinas y operaciones: por cuyo medio se hacen perceptibles a los que no las saben, ni profesan ${ }^{19}$.

Este homenaje a la acción iniciada por la Academia desde su fundación, unos veinte años antes, es también una justificación de la utilidad del aprendizaje del dibujo por todos los artesanos. Campomanes contestaba así a las críticas de algunas sociedades económicas, como la Matritense, que pedían una enseñanza más práctica de dibujos de máquinas en vez de la formación demasiado artística que se daba. Y es que la pedagogía impuesta por la Academia se fundaba en el modelo de las estatuas griegas que se utilizaba en sus clases de Madrid y en el principio, discutible sin duda, según el cual el que es capaz de dibujar las diferentes partes del cuerpo humano sin tenerlas a la vista sabrá dibujar cualquier máquina. Este principio pedagógico nos muestra que para la Academia artesanía y bellas artes formaban el conjunto inseparable de las «artes» y no podía pensarse la una sin la otra. Tal asociación, de inspiración neoclásica, correspondía a la voluntad de imponer a todos «el buen gusto» que evocaba el discurso de distribución de los premios de 1790, ya citado, cuando hablaba de «gentil y agradable manufactura» que debía ser una fuente de placer («nos causarán agrado y complacencia»). La primacía atribuida así a los principios de la estética neoclásica nos conduce a estudiar la dimensión propiamente artística del desarrollo de la Academia.

\section{La Academia como autoridad artística}

La doctrina estética de la Academia se caracterizó en sus primeros tiempos por cierta inconstancia debida a los artistas extranjeros llamados por los reyes

19 Rodríguez de Campomanes, Discurso sobre la educación popular de los artesanos, ed. de John Reeder, págs. 182-183. 
para dirigir las obras de decorado del Palacio Real de Madrid y del palacio de Aranjuez. Desde 1753 la responsabilidad de la enseñanza se atribuyó a tres artistas italianos representantes del arte barroco: el pintor Corrado Giaquinto, el escultor Olivieri y el arquitecto Sacchetti. Este dominio del barroco correspondía a la práctica de muchos pintores españoles de aquellos años, entre ellos José Luzán, pintor del rey a título supernumerario, en cuyo taller hizo Goya su aprendizaje en Zaragoza entre 1759 y 1763 . Pero la salida de Giaquinto que regresó a Italia en 1762 y la llegada a España de Mengs, llamado por Carlos III en 1761, provocaron un cambio radical. Las ideas estéticas de Mengs, el «pintor filósofo», apoyadas en los tratados teóricos de Winckelman, eran aprobadas unánimemente por la élite ilustrada de toda Europa. Negándose a sustraerse a la tutela extranjera, como algunos pedían, Carlos III atrajo a Mengs para favorecer la incorporación de los artistas españoles en la corriente estética dominante del tiempo. La adhesión a la doctrina de Mengs de varios artistas de la Academia, en particular su nuevo director Felipe de Castro y los arquitectos Villanueva y Ventura Rodríguez, convirtió pronto la institución en una plaza fuerte del neoclasicismo. Antes de llegar a ser Primer Pintor de Cámara en 1766, Mengs había sido nombrado en 1763 director honorario de la pintura de la Academia y él fue quien pidió la creación de las nuevas enseñanzas de anatomía, de perspectiva y de matemáticas. Después de su salida de España en 1777 la influencia de sus ideas siguió imponiéndose gracias a sus discípulos Salvador Maella y Francisco Bayeu, y gracias a un cenáculo de pensadores ilustrados reunidos alrededor de Antonio Ponz, que fue secretario de la Academia entre 1776 y 1790, y de Jovellanos que lo miraba como «el hijo de Apolo y de Minerva, el pintor filósofo, el maestro, el bienhechor y el legislador de las artes» ${ }^{20}$. Esta influencia también se extendió a los consiliarios, que definían la política artística de la institución y elegían a los nuevos académicos, a pesar de la ignorancia y de la ausencia de juicio artístico de algunos de ellos, denunciadas por Felipe de Castro, Goya y varios profesores, lo que confirma que los criterios de selección de los consiliarios eran más políticos que artísticos.

Las reglas neoclásicas impuestas por Mengs se convirtieron pues en una norma a la que debían someterse todos los candidatos a la Academia ${ }^{21}$. El mismo Goya tuvo que presentar un Cristo crucificado carente de la emoción y la violen-

20 Jovellanos, Elogio de las Bellas Artes (1781), citado por BEDAT, L'Académie des Beaux-Arts de Madrid., pág. 194.

${ }_{21}$ Sobre los debates en torno a la enseñanza de las bellas artes y la influencia de Mengs, véanse Esperanza Navarrete Martínez, La Academia de Bellas Artes de San Fernando y la pintura en la primera mitad del siglo XIX, Madrid, Fundación Universitaria Española, 1999; y Úbeda de los CoBos, Pensamiento artístico español del siglo XVIII. De Antononio Palomino a Francisco de Goya, Madrid, Museo Nacional del Prado, 2001. 
cia que caracterizaban las representaciones barrocas para ser admitido como «académico de mérito» en 1780. Pero ni este título, ni los cargos de profesor y luego de director adjunto de la Pintura que desempeñó a partir de 1785 impidieron que criticara severamente en una memoria redactada en 1792 la enseñanza que se impartía en la Academia. La autoridad que le daba su calidad de Pintor de Cámara desde 1789 le permitía expresarse con cierta libertad y denunciar el sistema establecido por Mengs, sin citar nunca su nombre. Todo su texto, que se abre con una crítica del autoritarismo mezquino practicado con los alumnos, es un alegato por la libertad de la enseñanza afirmada con fuerza en la conclusión:

Por último, Señor, yo no encuentro otro medio más eficaz de adelantar las artes, ni creo que le haya sino [...] el de dejar en su plena libertad correr el genio de los discípulos que quieren aprenderlas, sin oprimirlo, ni poner medios para torcer la inclinación que manifiestan a este o aquel estilo en la pintura ${ }^{22}$.

La libertad reivindicada por Goya no era sólo la autonomía respecto a las reglas gremiales que ofrecía la Academia a los artistas. Goya había podido darse cuenta, poco tiempo después de ser nombrado Académico de mérito, de los límites de esa libertad prometida por la Academia: encargado de decorar la capilla de la Virgen en la basílica del Pilar, tuvo que renunciar a su proyecto y someterse al plan previamente establecido por su cuñado, Francisco Bayeu, que gozaba de mucha autoridad como Pintor de Cámara del Rey y discípulo de Mengs. La libertad que Goya reivindicaba era precisamente la libertad de crear, fundada en el reconocimiento profesional del artista, la que él pedía cuando afirmaba en sus autorretratos su calidad de «Pintor», la que también reivindicaban en Francia en los mismos años los pensadores y escritores de las Luces, desde Voltaire y Diderot hasta d'Alembert y Rousseau ${ }^{23}$. Fue este deseo de libertad, más que sus problemas de salud, lo que le hizo renunciar a su cargo de director de la Pintura en 1797 para escapar de las obligaciones impuestas a los profesores de la institución y poder disponer del tiempo necesario para «emplearlo en cosas de mi gusto, que es de lo que carezco», como lo escribía a su amigo Zapater ${ }^{24}$. Pero Goya no llegó nunca a condenar explícitamente el sistema

22 Ángel Canellas, Francisco de Goya. Diplomatario, Zaragoza, Institución Fernando el Católico, 1981. Texto reproducido en apéndice de mi artículo «Goya y la enseñanza artística en la España del siglo XVIII", Miradas sobre une obra polifacética. Homenaje a Jean-Louis Guereña, Paris, Indigo, 2016, págs. 29-31.

${ }_{23}$ Sobre la lucha de Goya por la libertad y por la dignidad del artista, remito a mi libro Goya político, Madrid, Foro para el Estudio de la Historia Militar de España, 2013, en particular págs 42, 74-75.

24 Francisco de Goya. Cartas a Martín Zapater, ed. de Mercedes Águeda y Xavier de Salas, Madrid, Istmo, 2003, Carta de 2 de julio de 1788, pág. 278. 
de las academias, como los pensadores franceses que lo consideraban un obstáculo al reconocimiento del mérito individual. Por el contrario, las relaciones que siguió manteniendo, después de su dimisión de 1797, con una institución que lo nombró «Director honorario», atestiguan el papel de autoridad artística ineludible que ésta jugaba en la sociedad madrileña, a pesar de las dificultades financieras que limitaban su acción.

La escasez de créditos no le permitía en efecto a la Academia encargar muchas pinturas, y sólo lo hacía en circunstancias excepcionales. Una de ellas fue la obligación de adornar el salón de actos con un retrato del nuevo rey Fernando VII en marzo de 1808 y fue a Goya a quien se dirigió. El gran retrato ecuestre estaba terminado el 29 de marzo de 1808, pero ocho años después, Goya seguía pidiendo que se lo pagaran ${ }^{25}$.

Y fue también a Goya a quien pidió la Academia otro retrato ecuestre, en 1812, el de Wellington, que se exhibió en un salón de la calle de Alcalá entre el 2 y el 11 de septiembre de 1812, después de haber invitado el artista a toda la población madrileña a acudir a ver el retrato del vencedor de la batalla de los Arapiles. Pero esta vez Goya decidió donar su obra en homenaje al general victorioso.

La escasez de créditos no le permitía tampoco a la Academia comprar muchas obras de arte, pero tenía en depósito todo un conjunto de cuadros que habían sido confiscados con los bienes de los jesuitas y en el palacio de Godoy. Entre ellos se encontraban obras maestras de Rubens, del Tiziano, de Veronese, de Velázquez, de Murillo y de Van Loo, y también varios desnudos que no se podían exhibir. Todas estas obras permanecieron en los depósitos de la Academia hasta su traslado al nuevo museo del Prado en 1827. En cambio, en una época en que la capital no tenía ningún museo, la Academia organizó, primero cada tres años, luego cada año desde 1793, un «salón», a imitación de los que se celebraban en París desde 1737. En estas exposiciones madrileñas se presentaban al público las pruebas de examen de los nuevos académicos, además de las obras recientes de los profesores y de artistas cercanos a la institución. Goya se aprovechó de este «foro abierto», según la expresión de Esperanza Navarrete ${ }^{26}$, para inscribir en el espacio público unas obras que, de no ser así, sólo hubieran sido conocidas por el artista y su comanditario: entre 1795 y 1812 expuso en siete de esos «salones» anuales doce retratos, entre los cuales figuraban los de Francisco Bayeu (1795), Andrés del Peral (1798), Ferdinand Guillemardet

25 Canellas, Francisco de Goya. Diplomatario, cartas de 29 de marzo de 1808 y 14 de octubre de 1816.

26 Esperanza Navarrete Martínez, «Goya, la conveniencia de la Academia», Goya, la imagen inquieta, ed. Frédéric Prot, Presses Universitaires de Bordeaux, 2017, pág. 39. 
(1799), la marquesa de Villafranca e Isabel de Porcel (1805), Antonio Porcel (1806) e Isidoro Máiquez (1808). Estas exposiciones fueron las primeras que permitieron a un amplio público descubrir unas obras de arte que hasta entonces estaban reservadas para una élite social: el éxito que conocieron contribuyó mucho al resplandor de la Academia ${ }^{27}$.

Pero Goya no se contentó con participar en esas exposiciones anuales. Varias veces remitió obras suyas a la Academia para que las vieran y las juzgaran los profesores, aún cuando se apartaban de las normas neoclásicas. Fue lo que ocurrió en particular en 1794, después del grave accidente cerebrovascular que padeció, cuando mandó a Bernardo de Iriarte, Vice Protector de la Academia, once pequeños «cuadros de gabinete» como testimonio de su nueva manera de pintar «en que el capricho y la invención no tienen ensanches» ${ }^{28}$. Ya en 1797, el año en que dimitió de sus cargos académicos, pintó el retrato del mismo Bernardo de Iriarte, de quien alababa «la autoridad y la singular inteligencia».

Siguiendo las vivencias de Goya en sus relaciones con la Academia, traté de mostrar concretamente el papel que jugó ésta en la vida artística de la época. Después de un primer periodo de implementación reglamentaria bajo el reinado de Fernando VI, la Academia se desarrolló, diversificando sus enseñanzas y afirmando su identidad estética bajo la autoridad intransigente de Mengs. Goya fue el único artista que no aceptó el autoritarismo de la institución y dimitió de sus cargos conservando vínculos relativamente estrechos con ella. En conclusión, podemos decir que a lo largo de esos años la obra de Goya se fraguó contra la Academia, pero también con ella: contra su dogmatismo para afirmar los principios de lo que él llamó la «divina libertad» del hombre en general y del artista en particular. Pero también con ella, al mismo tiempo que ella se afirmaba como una autoridad ineludible, que obró por el reconocimiento social de las artes y de los artistas. El título de «Director honorario» que le había otorgado le permitió gozar de un trato privilegiado que se concretó en las obras que le encargó y las que expuso en los «salones» que organizó, lo que representa uno de los grandes méritos de la Academia: haber contribuido a promover en su tiempo la obra de un artista rebelde, cuando parte de esta obra, sin duda la más valiosa, quedaba censurada por el poder político e iba a ser condenada a un largo olvido que duraría hasta la década de 1870.

27 Sobre el éxito público de estas manifestaciones, véase la comunicación de Álvaro Molina MarTiN, «Las exposiciones de la Academia de San Fernando. Espacios y prácticas de saber artístico y sociabilidad» publicada en este mismo volumen de los Cuadernos de Estudios del siglo XVIII.

28 Canellas, Francisco de Goya. Diplomatario, Carta a Bernardo de Iriarte de 4 de enero de 1794, pág. 314 . 